\title{
An overview of chitosan and its application in infectious diseases
}

\author{
Qingye Meng ${ }^{1} \cdot$ Ying Sun ${ }^{1} \cdot$ Hailin Cong ${ }^{1} \cdot \mathrm{Hao} \mathrm{Hu}^{1} \cdot \mathrm{Fu}-J i a n \mathrm{Xu}^{2}$ (I)
}

Accepted: 18 January 2021 / Published online: 26 January 2021

(c) Controlled Release Society 2021

\begin{abstract}
Infectious diseases, such as the coronavirus disease-19, SARS virus, Ebola virus, and AIDS, threaten the health of human beings globally. New viruses, drug-resistant bacteria, and fungi continue to challenge the human efficacious drug bank. Researchers have developed a variety of new antiviral and antibacterial drugs in response to the infectious disease crisis. Meanwhile, the development of functional materials has also improved therapeutic outcomes. As a natural material, chitosan possesses good biocompatibility, bioactivity, and biosafety. It has been proven that the cooperation between chitosan and traditional medicine greatly improves the ability of anti-infection. This review summarized the application and design considerations of chitosan-composed systems for the treatment of infectious diseases, looking forward to providing the idea of infectious disease therapy.
\end{abstract}

Keywords Chitosan $\cdot$ Nanoparticle $\cdot$ Vaccine vector $\cdot$ Wound dressing $\cdot$ Infectious disease

\section{Introduction}

Infectious diseases are currently one of the main causes of human death. The prevention and treatment of these diseases have been received great attention. There was a period in history when the incidence and mortality of infectious diseases have been declining, which can be attributed to the development of penicillin [1]. However, the pace of drug development has fallen behind the microbial mutations rates, especially the emergence of super bacteria and new viruses [2]. The emergence of coronavirus disease-19 (COVID19) has brought a huge health crisis to all countries in the world. Seven coronaviruses infect humans currently, which including acute respiratory syndrome coronavirus (SARS-CoV) in 2002, Middle East respiratory syndrome coronavirus (MERS-CoV) in 2012, and the COVID-19 or

$\mathrm{Hao} \mathrm{Hu}$

huhao@qdu.edu.cn

$\triangle$ Fu-Jian Xu

xufj@mail.buct.edu.cn

1 Institute of Biomedical Materials and Engineering, College of Materials Science and Engineering, Qingdao University, Qingdao 266071, China

2 Key Lab of Biomedical Materials of Natural Macromolecules, Ministry of Education, Beijing Laboratory of Biomedical Materials, Beijing University of Chemical Technology, 100029 Beijing, China named SARS-CoV-2 last year [3, 4]. The development of new drugs and vaccines cannot be delayed [5]. Meanwhile, effective treatment strategies are constantly being tried in the clinic. In recent years, the dynamics of infectious diseases and the emergence of drug-resistant bacteria and viruses have also made the biomedical field pay more attention to new drug delivery platforms.

Most infectious diseases are caused by bacteria, fungi, parasite, and viruses [6]. The current therapy methods for infectious diseases include vaccine injection and drug treatment for the infected site, which ultimate goal is to eliminate pathogens. Most vaccines are injected parenterally to stimulate the immune system to produce antibodies in the serum, but cannot produce mucosal antibody responses [7]. In recent years, needle-free administration via oral, nasal, pulmonary, and dermal routes has become an alternative to parenteral administration [8]. Some drug delivery systems based on micro- or nano-particles have been developed for targeted delivery of vaccines to generate effective adaptive immunity. Because of the high specific surface area of nanoparticles, drug molecules can obtain ideal drug loading efficiency in particles [9]. Vectors with low cost, good biocompatibility, and low side effects have attracted the interest of researchers.

Chitosan is a natural and biocompatible polysaccharide, mainly derived from the exoskeleton of crustaceans. Due to the presence of quaternary ammonium salt groups, chitosan 
exhibits positive charges in physiological environments. [10]. A large number of active functional groups on the molecular chain make chitosan easy to be structurally and chemically modified to produce immune stimulation [11], the promotion of wound healing [12], and antibacterial and antifungal properties [13]. The versatility and adaptability of chitosan provide a unique opportunity for the development of new antibacterial therapies and prevention of infectious diseases. Chitosan-composed systems utilize the characteristics of chitosan to achieve great therapeutic effects. For example, the adhesiveness of chitosan can be used for noninvasive mucosal vaccine vectors [14]. When chitosan combined with other wound dressings such as hydrogels, it has certain antibacterial properties while promoting wound healing [15]. Chitosan-modified vectors exhibit good biocompatibility in vivo. Therefore, chitosancomposed systems have great potential in the therapy of infectious diseases.

This review summarized the application of chitosan in the treatment of infectious diseases. By analyzing its design considerations and application, new ideas for the therapy of infectious diseases are expected to be proposed.

\section{The application of chitosan in the infectious diseases therapy}

The antibacterial action mechanism of chitosan is to bind to the negatively charged bacterial cell wall, which leads to a change in the permeability of the cell envelope and then attaches to the DNA to inhibit its replication [16]. The physical and biological properties of chitosan depend on the degree of deacetylation and molecular weight [17]. The number and distribution of acetyl groups in chitosan affect its biodegradability and cytotoxicity, and the degree of deacetylation of chitosan directly affects the efficiency of antigen delivery and the activity of chitosan as an adjuvant $[18,19]$. The amine and hydroxyl functional groups present in chitosan can be chemically modified to introduce other groups, such as hydroxyalkyl [20,21], carboxyalkyl [22], succinyl [23], thiol [24, 25], and sulfate [26], to prepare various chitosan derivatives. The adhesion and permeability enhancement of chitosan derivatives depend on the degree of substitution or quaternization of chitosan.

The construction of chitosan-composed nanoscale vectors can take advantage of the size to achieve great therapeutic effects. There are various kinds of delivery systems, not limited to nanocarriers, and corresponding requirements for delivery systems are also varied. For instance, nanoparticles as drug delivery systems need to responsive release characteristics and require good biocompatibility; meanwhile, wound dressings emphasize the mechanical properties of the material to achieve sustained drug release.
Hydrogels need to have good biocompatibility and the promotion of wound healing ability. Hydrogels loaded with therapeutic drugs have been proven to promote wound repair or enhance antibacterial and anti-infective properties. Therefore, in response to different infectious diseases, the treatment strategies tend to be diversified.

\section{Chitosan-composed nanoparticles}

Chitosan and its derivatives have antibacterial effects on Gram-negative bacteria and Gram-positive bacteria [27]. The effectiveness of the antibacterial agent on bacteria can be improved by being loaded into the chitosan-composed nanoparticles [28]. Chitosan can also work in collaboration with other nanoparticles. Nanoparticles have a high specific surface area. The high surface area leads to a higher charge density, thereby increasing the interaction with microbial elements [13]. For instance, chitosan films were incorporated onto gold nanoparticles to achieve improved antibacterial effects [29] (Fig. 1). The intrinsic effects of chitosan-composed nanoparticles on microorganisms have been enhanced on the nanoscale, while the adverse effects have hardly increased [30-32]. Chitosan-composed nanoparticles showed obvious antibacterial effects on $E$. coli and several Staphylococcus [13]. These chitosancomposed nanoparticles are superior to pure chitosan polymers and doxycycline in inhibiting the growth of these species. Friedman et al. studied the antibacterial and immunological properties of chitosan-sodium alginate nanoparticles, which showed effective inhibition of the growth of $P$. acnes [33]. Studies have shown that chitosan nanoparticles, especially those formed with low molecular chitosan, can inhibit the formation of Streptococcus mutans biofilm in vitro [34]. Chitosan-composed nanoparticles can be uniformly dispersed throughout the sample and cause significant cell membrane damage [31]. Similarly, the antifungal mechanism of chitosan-composed nanoparticles is also through the close interaction of chitosan and fungal cell membranes, which caused fungal cell membrane rupture [35]. But it should be noted that $A$. niger is resistant to chitosan nanoparticles [36]. The cell wall of $A$. niger contains $10 \%$ chitin, which is related to its resistance to chitosan. Therefore, chitosan-composed nanoparticles need specific choices in antifungal aspects to achieve the ideal therapeutic effect.

Helicobacter pylori can cause gastroesophageal reflux disease and chronic atrophic gastritis [37]. The drug resistance of $H$. pylori has increased sharply, and the efficacy of traditional antibiotics has been severely reduced. Chitosan nanoparticles can be used for gastric delivery and continuous administration [38]. This strategy can overcome malabsorption of the drug in the stomach. Chitosan nanoparticles can also be used for ocular 


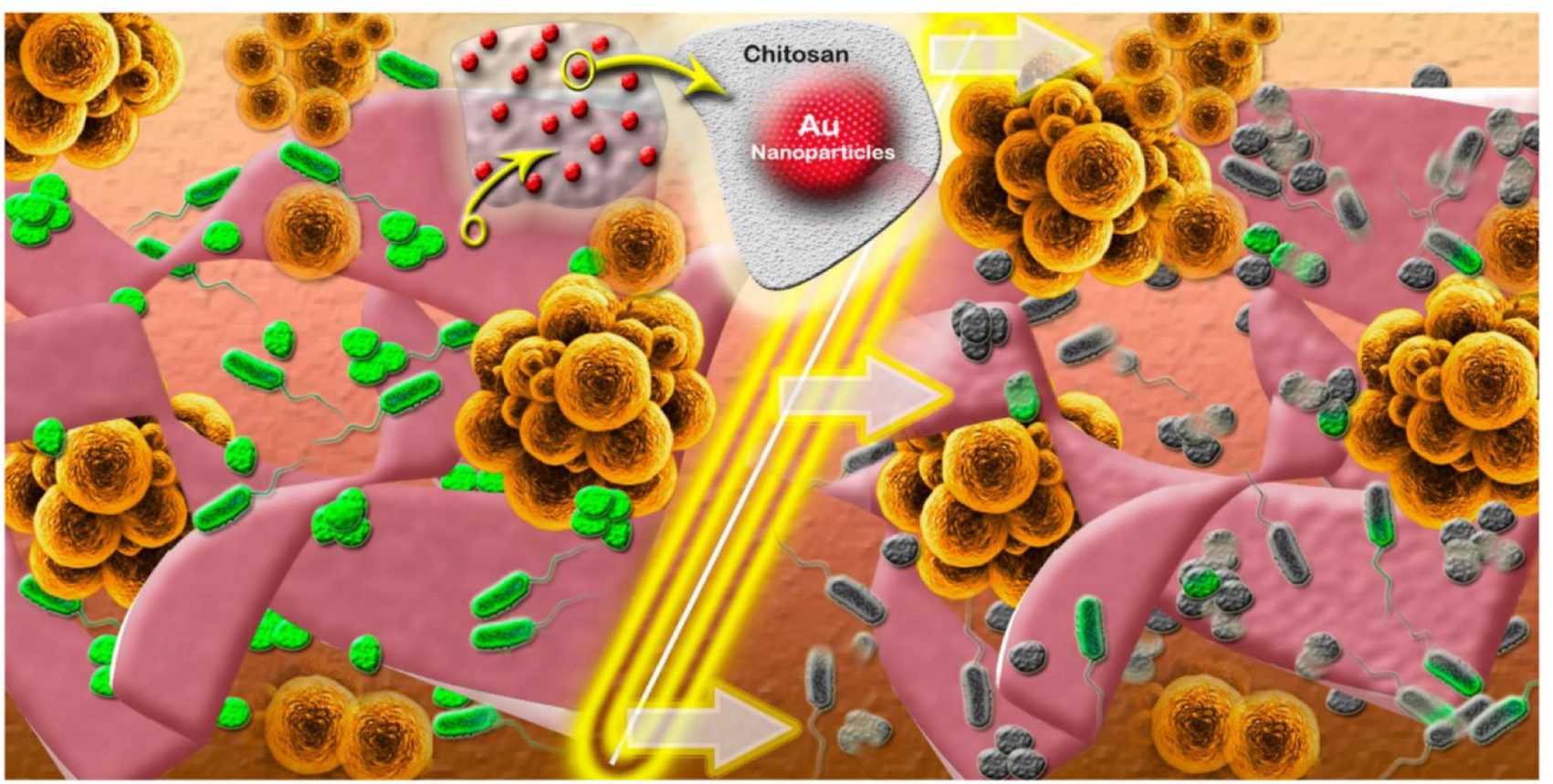

Fig. 1 Schematic diagram of chitosan-gold nanoparticles as efficient antibacterial materials [29]. Copyright (C) 2014 American Chemical Society

administration. Zhou et al. designed chitosan-modified polylactic acid nanoparticles for ocular drug delivery [39] (Fig. 2). Compared with the free drug, with the same antibacterial properties, this drug delivery system exhibits better corneal penetration without increasing corneal irritation. These advantages promoted the strategic design of chitosan-composed nanoparticles in drug delivery systems. The biodegradable nano-delivery system that combined polysaccharides and proteins has also attracted interest of researchers. There are reports on the preparation of nanoparticles by electrostatic complexation of proteins/ polysaccharides [40, 41]. The stability of embedding agent and nanoparticles can be improved by increasing the spatial and electrostatic repulsion between protein and polysaccharide. The stability of nutrient compounds can be greatly improved by encapsulating hydrophobic and hydrophilic nutritive compounds into protein/ polysaccharide electrostatic complexes [42]. A kind of vector modified by corona with oppositely charged macromolecules has been reported [43]. For example, proteins act as the inner core to change the overall particle density and provide sustained release of drugs; meanwhile, polysaccharides act as the outer layer to provide additional steric and electrostatic stability $[42,43]$. The biodegradable nanoparticles were corona-modified by chitosan, and the adhesion of biodegradable nanoparticles was improved through electrostatic interaction with the anionic mucus layer. This proposal exhibited a good therapeutic effect on H. pylori in the gastric epithelial layer and the mucus layer. To sum up, it is of significance for improving the curative effect to construct a nano-carrier based on chitosan or to modify the carrier with chitosan.
Fig. 2 Schematic diagram of self-aggregation formed by the PLA- $g$-CS copolymer in aqueous medium [39]

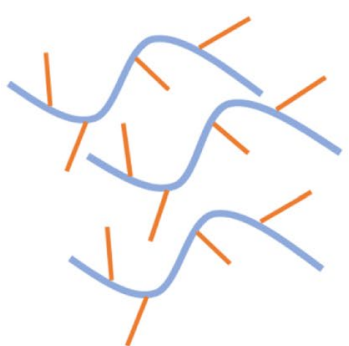

PLA-g-CS copolymer

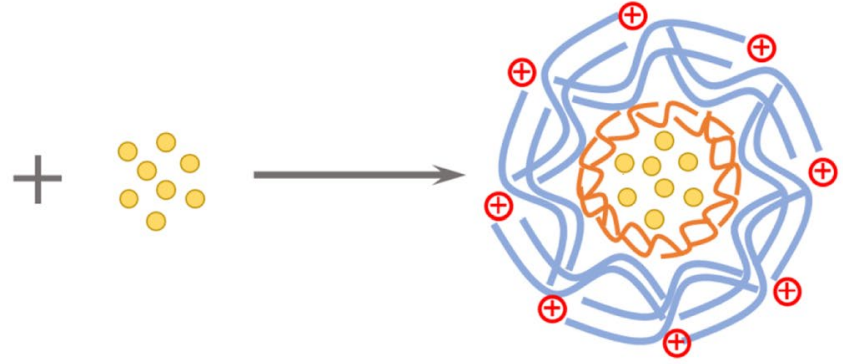

AmB
AmB-incorporated nanoparticle 


\section{Chitosan-based vaccine vectors}

For the prevention and treatment of infectious diseases, drug treatment often brings some undesirable side effects and increases the drug resistance of bacteria and viruses. Vaccination is one of the effective treatment methods for the prevention and treatment of infectious diseases. By activating the immune system, the recurrence of infectious diseases can be effectively prevented [44]. The mucosal surface (nose, respiratory tract, oropharynx, gastrointestinal tract, and genitourinary system) is the most common entry route for pathogens (viruses and bacteria) [45]. Mucosal immunity not only provides humoral and cellular immune protection in the mucosal area but also provides systemic immunity [46]. Polymer-based vectors have flourished in mucosal vaccine delivery because they provide the advantage of delivering antigens to specific targets [47]. Moreover, the polymer-based vector can protect the antigen from harsh environments such as $\mathrm{pH}$, bile, and gastrointestinal tract digestive enzymes, as well as can control or slow the release of the antigen. It has been reported that polymer-based particles can enhance the immune response to mucosal delivery of antigens [48] (Fig. 3). After encountering with an antigen, B cells convert themselves to antibody secreting plasma cells that produce antibodies for excreting the pathogens to mucosal surfaces (mucosal response), whereas dendritic cells (DCs) present the antigen via major histocompatibility complex (MHC) class I and class II molecules to CD8+ and CD4+ T cells. The activation pathway of CD8+ T cells and CD4+ Th1 cells produces cytotoxic $\mathrm{T}$ lymphocytes (CTL) and activated macrophages that kill intracellular pathogens or infected cells (cellular response), while the activation pathway of $\mathrm{CD} 4+\mathrm{Th} 2$ cells produces activated B lymphocytes that secrete antibodies for neutralization of extracellular pathogens (humoral response).

Considering the optimal performance of the polymerbased vector and the required conditions for the delivery of antigens through the mucosal route, chitosan and its derivatives have attracted great attention as the particle vector for antigen delivery. The adhesion of chitosan is mainly caused by the electrostatic interaction between the positively charged molecular chain and the negatively charged cell surface and mucus [49]. Subbiah et al. loaded hepatitis B virus surface antigen (HBsAg) into $N, N, N$ trimethyl chitosan nanoparticles (TMC NPs) for controlled intranasal administration (Fig. 4) [50]. In vivo immune studies shown that the adjuvant efficiency of antigen-loaded
Fig. 3 Schematic diagram of various immune responses induced by particulate vaccine system [48]. Copyright (C) 2017 Elsevier B.V

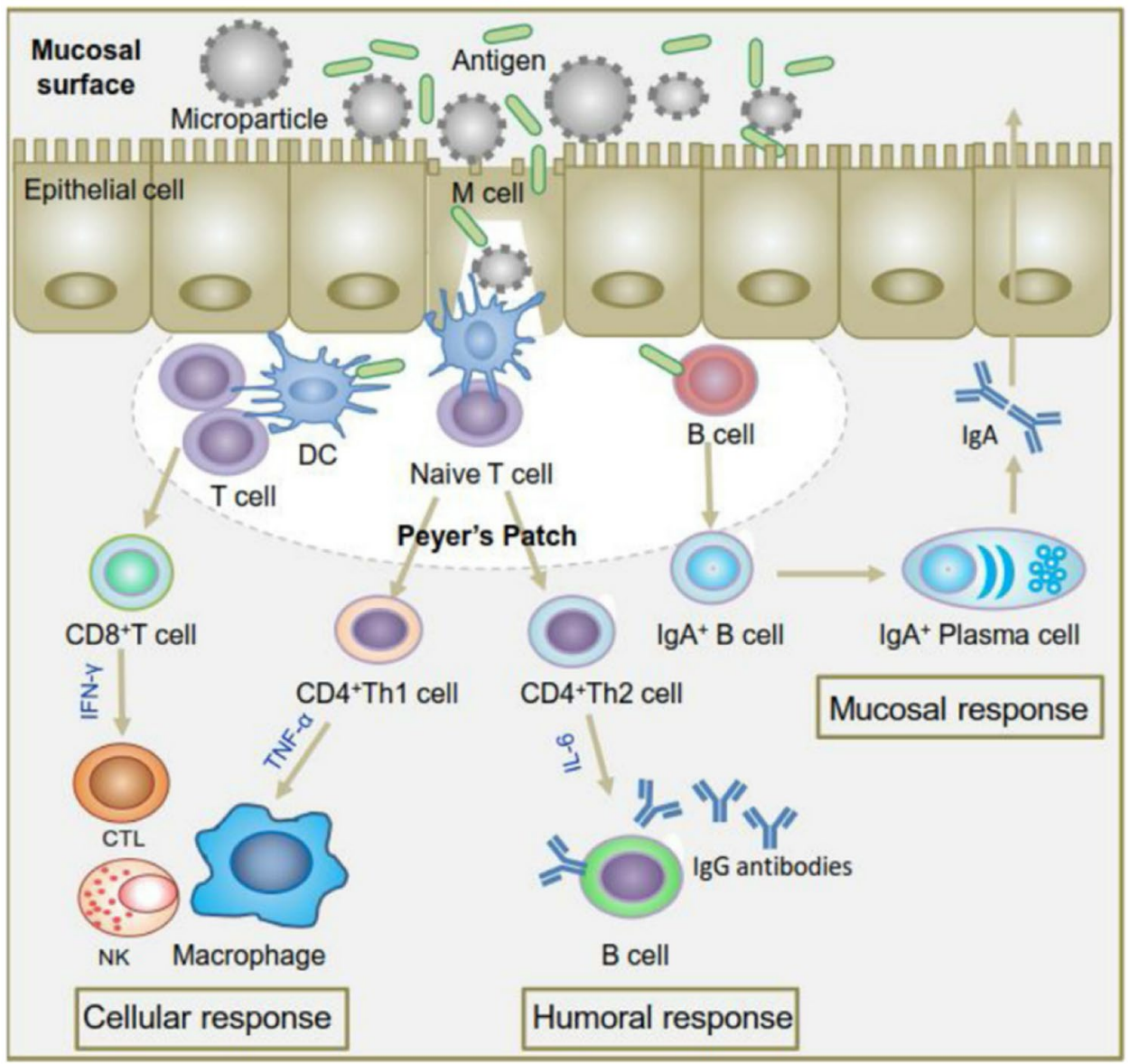


Fig. 4 Schematic diagram of $\mathrm{N}, \mathrm{N}, \mathrm{N}$-trimethyl chitosan (TMC) NP preparation, HBsAg loading, and nasal administration of TMC NPs [50]. Copyright (C) 2012 Elsevier Ltd

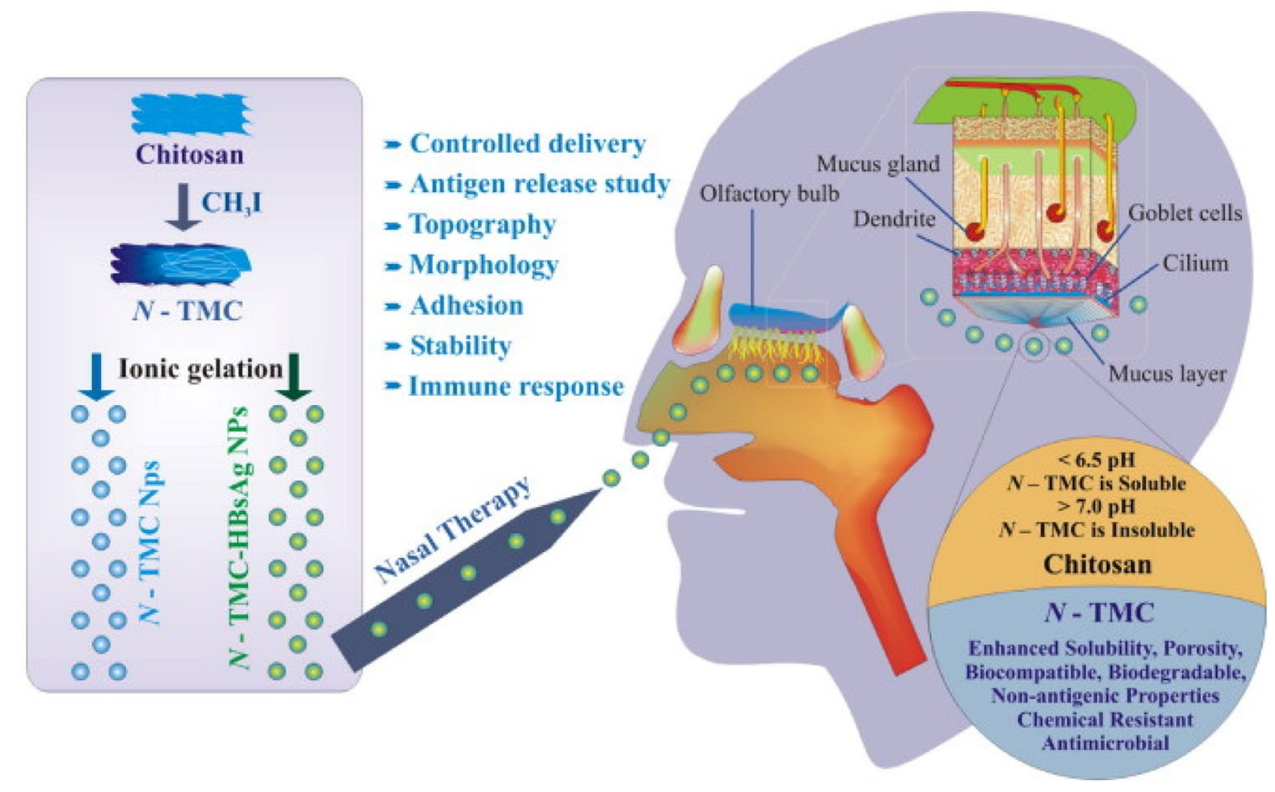

TMC NPs remains highly stable for a long period of time. However, it is worth noting that the aggregation behavior of chitosan particles affects the long-term stability of vectors. PEGylation can enhance the stability of the vector during circulation in vivo [14]. PEGylated chitosan microspheres can release more antigens in vitro and stimulate macrophages to produce more cytokines. Pluronic as a typical multi-purpose excipient has been widely used in various pharmaceutical preparations due to its ability to increase water solubility and drug stability. It has been reported that the two-component system of Pluronic F127 and chitosan showed additive or synergistic effects in immune response [51].

Vaccine vectors modified with targeting ligand can greatly improve the delivery efficiency of the antigen [52]. Chitosan surface-modified by mannose has been used as nasal delivery of vaccines [53]. Similarly, targeting peptides conjugated to chitosan can also improve the targeting efficiency of vaccine vectors [54]. Chitosan, as a positively charged polysaccharide, shows great advantage in constructing vaccine vector.

\section{Chitosan-based wound dressings}

Local infections are widespread in wounds, burns, diseases, surgical wounds, and all other tissues involved in surgery. A local infection may lead to prolonged wound healing, wound dehiscence, abscess formation, and sepsis, and in severe cases, it can also lead to life-threatening complications [55]. Therefore, the development of new biological materials that can prevent infection has become an urgent and important goal. Due to antibacterial properties, chitosan has inherent advantages as wound dressings. In the environment of multivalent electronegative molecules or anions, chitosan can form ion network through coordination and secondary interactions, which form gel network easily [56]. In recent years, materials with three-dimensional network composition have received more and more research as a new type of vector platform, such as three-dimensional scaffolds, drug banks, bandages, and wound dressings [57-59]. It is worth noting that biomedical hydrogels based on natural polymers, especially chitosan, have received special attention due to their good biocompatibility and biomimetic properties [60]. Chitosan-based gels can encapsulate biologically active molecules in the network through physical interaction or chemical coupling. For example, a hydrogel composed of carboxymethyl chitosan (CMC)/oxidized dextran was developed for loading anti-infective drugs (ceftriaxone sodium) [61]. The prepared hydrogel has been proven to have good biocompatibility and exhibit good anti-infective effects in vivo (subcutaneous infection model and cecal ligation and perforation model). $\mathrm{Hu}$ et al. used CMC, alginate (ALG), and calcium chloride to prepare a series of double-crosslinked amorphous hydrogels (CMC-ALG) through electrostatic interaction and divalent chelation, which loaded epidermal growth factor (EGF) (Fig. 5) [62]. The double cross-linked amorphous hydrogel promotes wound healing while being antibacterial.

As shown in Fig. 6, Yang et al. developed a highperformance hybrid chitosan-polyacrylamide (CS-pam) ion covalent double-network hydrogel and flexibly adjusted its structure and mechanics by adjusting the CS ion network in situ [63]. The application of this hydrogel in wound dressings has great prospects.

Chen et al. fabricated antibacterial alginate/chitosan hydrogel dressing integrated with gelatin microspheres. 


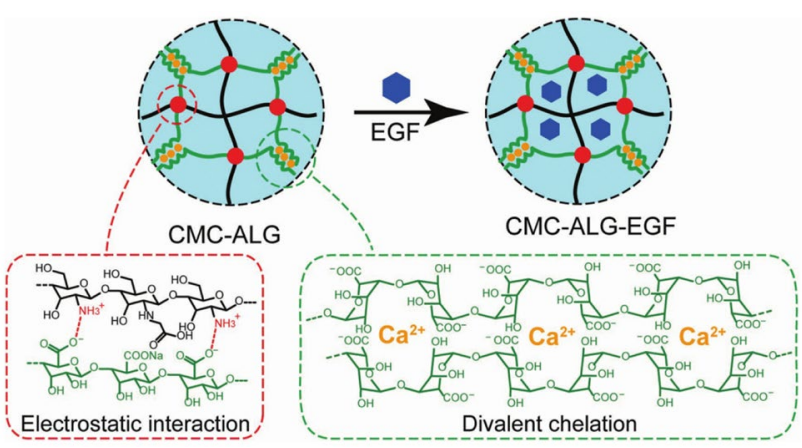

Fig. 5 Schematic illustration of CMC-ALG and CMC-ALG-EGF hydrogels [62] Copyright (C) 2018 WILEY- VCH Verlag GmbH \& Co. KGaA, Weinheim.

Drug-loaded gelatin microspheres improved the biodegradable and mechanical properties of the dressing. The composite dressing showed antibacterial performance and bacterial growth inhibition effects [64]. Bandages with hemostatic and antibacterial effects were prepared by using chitosan acetate [65]. Compared with alginate sponge bandage and silver sulfadiazine cream, the chitosan acetate bandages can reduce the level of bacterial load in the skin more efficiently. In the $P$. aeruginosa and $P$. mirabilis models treated with chitosan acetate bandages, systemic infections can be prevented, and all animals can survive.

\section{Design considerations for chitosan-based drug delivery systems}

Chitosan component can improve the biocompatibility, biosafety, and stability of drug delivery systems via chemical modification or physical mixing. According to different treatment strategies, the expected performance of the vector is diverse. For example, drug vectors need controlled release function, mucosal vaccine carriers need adhesive properties, and wound dressings require certain physical properties.

\section{Adhesion}

In mucosal vaccine carriers, chitosan has been used to increase the adhesive ability of vaccine carriers to achieve long-term retention on the mucosa [49]. In the antibacterial therapy of dental diseases, insufficient absorption and penetration of biofilms by traditional antibacterial agents are two major considerations for the use of these therapies [66]. Biofilms are complex bacterial communities that are highly resistant to antibiotics and human defense systems [67]. Researchers have done plenty of work on the antibiofilm/antibacterial potential of metals and metal oxide nanoparticles, such as titanium oxide, copper, silver, gold, zinc, and silicon [68]. However, the side effects of metal nanoparticles limited their clinical applications. Therefore, natural biopolymers with adhesive properties can be used as potential antibacterial and anti-inflammatory nano-drug delivery systems $[69,70]$. Chitosan has adhesive properties and is used to treat periodontitis. A faster healing rate of alveolar bone and periodontal epithelium in mice with periodontitis was observed [71]. The vector system can be attached to the tooth surface, tongue surface, and buccal cavity mucosa. As the environment of the oral cavity changes, the antibacterial agent can be released in response, so the antibacterial agent can penetrate the bacterial biofilm that caused the dental infection to achieve a therapeutic effect.

\section{The balance between efficiency and toxicity}

Polysaccharides have received extensive attention in the field of pharmacy due to their high-efficiency therapeutic applications in drug storage and reducing toxicity [72]. Almeida et al. prepared chitosan-based amphiphilic micelles for hydrophobic drug delivery [73]. This delivery strategy has demonstrated that chitosan micelles can stabilize the loaded drug, protect up to $75 \%$ of the drug from hydrolysis and retain its activity. The antibiotic polymyxin $\mathrm{B}$ (PMB) is considered to be an effective weapon against gram-negative
Fig. 6 Fabrication of hybrid CS-PAM ionic-covalent doublenetwork hydrogels [63]. Copyright (C) 2018 WILEY-VCH Verlag $\mathrm{GmbH} \& \mathrm{Co} . \mathrm{KGaA}$, Weinheim

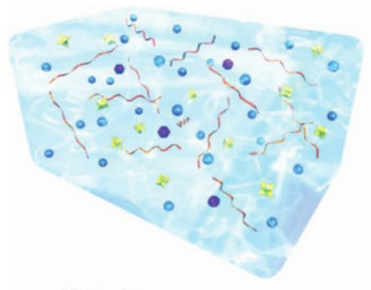

Solution

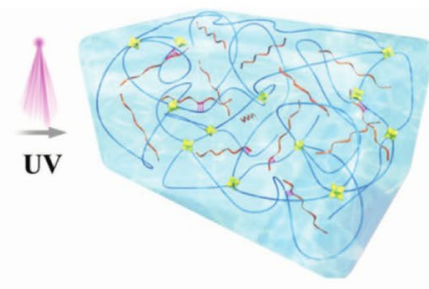

Composite Hydrogel

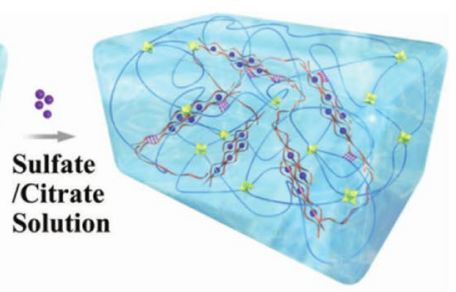

DN Hydrogel
- Acrylamine

Photoinitiator

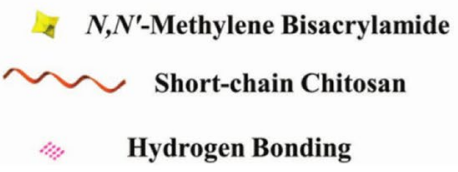


"super bacteria" [74]. However, the severe nephrotoxicity and neurotoxicity of PMB limits its application [75, 76]. There have been many methods to reduce the toxicity of polymyxin by shielding the cationic part or reducing the number of cationic residues by negatively charged derivatives. For example, PMB prodrugs prepared from cationic amino acids modified by methanesulfonic acid and PEG [77, 78]. Although PMB prodrugs can significantly reduce its toxicity, it inevitably reduces its antibacterial properties. Some traditional drug delivery nano-platforms such as liposomes [79], silica nanoparticles [80], and polymer particles [81, 82] can improve the safety of PMB. However, due to the restriction of entering the bacterial membrane, the bactericidal activity of these nanocarriers is reduced. Chai et al. developed a pH-sensitive multi-ion nanocomplexes to deliver PMB for the treatment of acute lung infections, which showed fewer adverse reactions without affecting the inherent antibacterial effect (Fig. 7) [83]. This multi-ionic nanocomplexes are mixture of PMB and 2,3-dimethylmaleic anhydride (DA) grafted chitoligosaccharide. As a derivative of chitosan, chitoligosaccharide can be excreted or biodegraded in the body. By electrostatically complexing with negatively charged DA, the positive charge density of PMB was reduced, which leads to the decline of the toxicity. This delivery system showed the same antibacterial properties as free PMB in vivo, but significantly reduced the nephrotoxicity and neurotoxicity caused by PMB. Moreover, this method of electrostatically combining antibiotics and biocompatible materials can be extended to other antibiotics as a general strategy to reduce the side effects of highly toxic antibiotics.

\section{Combination therapy}

Chitosan have great potential in combination therapy. Whether through chemical modification or physical mixing, the biocompatibility, biosafety, and stability of the drug delivery system could be improved. In some cases, the combination of chitosan and other therapeutic drugs can produce synergistic therapeutic effects.
Chitosan is an attractive preparation widely used as a pharmaceutical excipient. El-Sharif et al. explored the antibacterial activity of the chitosan-EDTA combination against gram-negative bacteria, gram-positive bacteria, and Candida albicans [84]. This research showed that the use of chitosan as an enhancing agent with antibacterial and antifungal properties in combination with EDTA in pharmaceutical preparations. Camacho-Alonso et al. explored the antibacterial effect of chitosan accompanied by photodynamic therapy (PDT) on Enterococcus faecalis and evaluated the enhancement effect of chitosan on the photosensitizer methylene blue in the root canals of extracted human teeth infected in vitro [85].

Some matrixes can be chemically or physically modified by chitosan, which achieve good biocompatibility and antibacterial properties. The permanent presence of metal vascular stents in the blood vessel wall can cause foreign body reactions and thrombosis [86]. Researchers have developed bioabsorbable vascular stents (BVS) to overcome the above-mentioned limitations of metal stents. BVS can be absorbed over time without any permanent prosthetic constraints in the blood vessel [87]. Poly(Llacticacid) (PLLA) is currently the most commonly used BVS polymer [88]. But compared with permanent metal stents, BVS made of PLLA faces a higher incidence of early thrombosis [89]. To solve this problem, chitosan is used to form heparin-like polysaccharide multilayer film with sulfated yam polysaccharide (SCYP) to assemble and modify the surface of PLLA membrane layer by layer [90]. The multilayer modified PLLA prepared by this strategy is non-cytotoxic, has good antibacterial activity against $E$. coli and $S$. aureus, and has drug loading/sustained release behavior. This strategy has potential applications in biomedical materials that contact blood. The combination of chitosan and antibacterial drugs provides an idea for constructing versatile treatment systems. These therapy strategies were summarized in Table 1, which not limited to the treatment of infectious diseases, which had a certain inspiration for the treatment of other diseases.
Fig. 7 Schematic illustration of preparation process of CS-DA/ PMB nanocomplexes [83]. Copyright $\odot 2020$ WILEYVCH Verlag $\mathrm{GmbH} \& \mathrm{Co}$. $\mathrm{KGaA}$, Weinheim

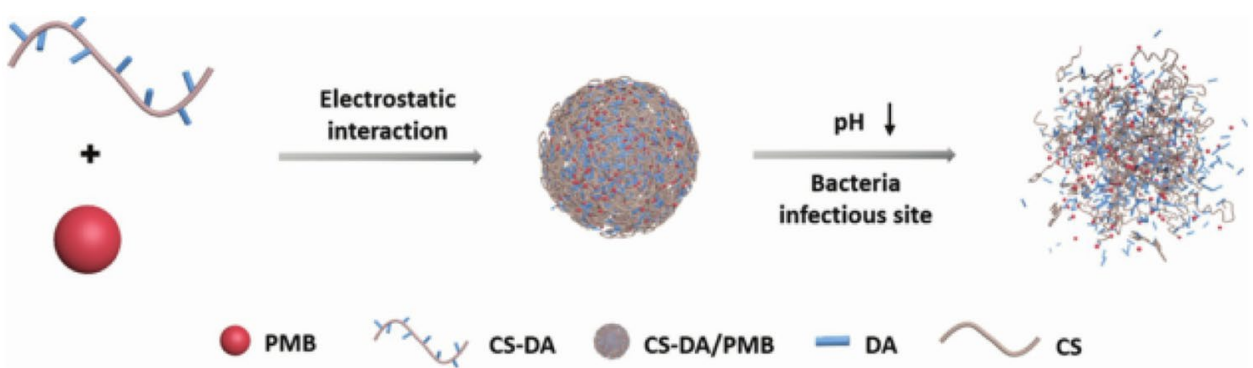


Table 1 Summary of chitosanbased vectors in drug delivery

\begin{tabular}{llll}
\hline Vector type & Application & Design considerations & Ref \\
\hline Nanoparticles & Antibacterial & Antibacterial & {$[33]$} \\
Nanoparticles & Anti-fungal & Anti-fungal mechanism & {$[35]$} \\
Nanoparticles & Drug delivery & Continuous administration & {$[38]$} \\
Nanoparticles & Ocular administration & Corneal penetration & {$[39]$} \\
Nanoparticles & Vaccine vectors & Stability and adhesion & {$[50]$} \\
Hydrogel & Wound dressings & biocompatibility & {$[62]$} \\
Hydrogel & Wound dressings & Mechanical property & {$[63]$} \\
Hydrogel & Wound dressings & Biodegradable and mechanical & {$[64]$} \\
& & properties & \\
Hydrogel & Periodontitis & Adhesive property & {$[71]$} \\
Micelles & Drug delivery & Stability & {$[73]$} \\
Nanocomplexes & Drug delivery & Biocompatibility & {$[83]$} \\
Complexes & Antibacterial & Combination therapy & {$[84]$} \\
Complexes & Antibacterial & Combination therapy & {$[85]$} \\
Multilayer film & Antibacterial and Drug delivery & Combination therapy & {$[90]$} \\
\hline
\end{tabular}

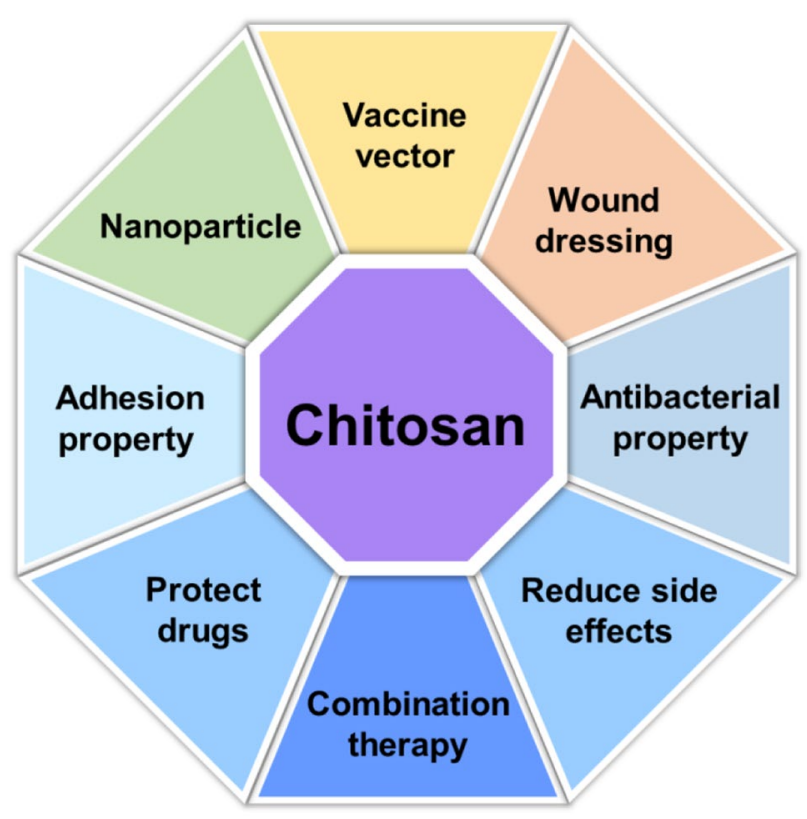

fungi, and viruses that cause infectious diseases. But the biocompatibility of chitosan can be applied to the human body. Due to the low-cost and highly biocompatible, chitosan-based vectors have great potential in the future.

As summarized in Fig. 8, in terms of infectious diseases, chitosan-composed systems in different forms or with diverse properties have been proposed. Chitosan-composed vectors have great application potential in vaccine vectors and drug vectors. These kinds of vectors can efficiently load therapeutic drugs, reduce the toxic and side effects of the drugs, and enhance the treatment efficacy. These concepts have certain potential in the development of clinical applications. In addition, due to its good antibacterial ability and biocompatibility, chitosan-based wound dressings have good prospects in clinical applications. As the wound dressing, it is not only required to promote wound healing, but also should be dedicated to rapid analgesia, anti-itching, and alleviating the discomfort of patients during the healing process. Moreover, simplifying the preparation steps of chitosan-based dressings can also promote its development in clinical applications. For the current epidemic of COVID19 and possible viruses in the future, chitosan as a vaccine vector has high application value in terms of non-invasive vector. The chitosan-based vector can deliver antibodies to the nasal mucosa, and can load gene drugs that interfere with or disrupt viral DNA/RNA replication and deliver them to infected cells. These provide some inspiration for the fight against COVID-19 in the future.

The development of new vectors increases the diversity of combination with chitosan-composed systems. It is believed that in the near future, more chitosan-composed systems would be developed in the treatment of infectious diseases and be widely used in clinical practice. 
Funding This work is financially supported by the National Natural Science Foundation of China (51703105 and 51829301), Natural Science Foundation of Shandong Province (ZR2017BEM012), Beijing Outstanding Young Scientist Program (BJJWZYJH01201910010024), Fundamental Research Funds for the Central Universities (XK1802-2), and China Postdoctoral Science Foundation (2018M630752).

\section{Declarations}

Competing interests The authors declare that they have no competing interests.

\section{References}

1. Gupta A, Kumar S, Kumar R, Choudhary AK, Kumari K, Singh P, et al. COVID-19: Emergence of infectious diseases, nanotechnology aspects, challenges, and future perspectives. Chemistry Select. 2020;5(25):7521-33. https://doi.org/10.1002/ slct.202001709.

2. Huh AJ, Kwon YJ. "Nanoantibiotics": a new paradigm for treating infectious diseases using nanomaterials in the antibiotics resistant era. J Controlled Release. 2011;156(2):128-45. https:// doi.org/10.1016/j.jconrel.2011.07.002.

3. Zhong NS, Zheng BJ, Li YM, Poon LLM, Xie ZH, Chan KH, et al. Epidemiology and cause of severe acute respiratory syndrome (SARS) in Guangdong, People's Republic of China, in February, 2003. The Lancet. 2003;362(9393):1353-8. https://doi. org/10.1016/S0140-6736(03)14630-2.

4. Zaki AM, van Boheemen S, Bestebroer TM, Osterhaus ADME, Fouchier RAM. Isolation of a novel coronavirus from a man with pneumonia in saudi arabia. N Engl J Med. 2012;367(19):1814-20. https://doi.org/10.1056/NEJMoa1211721.

5. Chen B, Tian EK, He B, Tian L, Han R, Wang S, et al. Overview of lethal human coronaviruses. Signal Transduction and Targeted Ther. 2020;5(1):89. https://doi.org/10.1038/s41392-020-0190-2.

6. Lashley FR. Emerging infectious diseases: vulnerabilities, contributing factors and approaches. Expert Rev Anti-Infect Ther. 2004;2(2):299-316. https://doi.org/10.1586/14787210.2.2.299.

7. Senel S. Chitosan-based particulate systems for non-invasive vaccine delivery. Adv Polym Sci. 2011;243:111-37. https://doi. org/10.1007/12_2011_120.

8. Lambert PH, Laurent PE. Intradermal vaccine delivery: Will new delivery systems transform vaccine administration? Vaccine. 2008;26(26):3197-208. https://doi.org/10.1016/j.vaccine. 2008.03.095.

9. Kim BY, Rutka JT, Chan WC. Nanomedicine. N Engl J Med. 2010;363(25):2434-43. https://doi.org/10.1056/NEJMra0912273.

10. Kong M, Chen XG, Xing K, Park HJ. Antimicrobial properties of chitosan and mode of action: a state of the art review. Int $\mathrm{J}$ Food Microbiol. 2010;144(1):51-63. https://doi.org/10.1016/j. ijfoodmicro.2010.09.012.

11. Gorbach VI, Krasikova IN, Luk'yanov PA, Loenko YN, Solov'eva TF, Ovodov YS, et al. New glycolipids (chitooligosaccharide derivatives) possessing immunostimulating and antitumor activities. Carbohydr Res. 1994;260(1):73-82. https://doi. org/10.1016/0008-6215(94)80023-5.

12. Madhumathi K, Sudheesh Kumar PT, Abhilash S, Sreeja V, Tamura H, Manzoor K, et al. Development of novel chitin/ nanosilver composite scaffolds for wound dressing applications. J. Mater. Sci.: Mater. Med. 2010;21(2):807-13 https://doi. org/10.1007/s10856-009-3877-z.

13. Qi L, Xu Z, Jiang X, Hu C, Zou X. Preparation and antibacterial activity of chitosan nanoparticles. Carbohydr
Res. 2004;339(16):2693-700. https://doi.org/10.1016/j.carre s.2004.09.007.

14. Jiang HL, Park IK, Kang ML, Yoo HS, Choi YJ, Akaike T, et al. Immune stimulating activity of an atrophic rhinitis vaccine associated to pegylated chitosan microspheres in vitro. Polym Adv Technol. 2007;18(3):220-5. https://doi.org/10.1002/pat.861.

15. Liu XF, Guan YL, Yang DZ, Li Z, Yao KD. Antibacterial action of chitosan and carboxymethylated chitosan. J Appl Polym Sci. 2001;79(7):1324-35. https://doi.org/10.1002/1097-4628(20010214) 79:7\%3c1324::AID-APP210\%3e3.0.CO;2-L.

16. Chakraborty SP, Sahu SK, Pramanik P, Roy S. In vitro antimicrobial activity of nanoconjugated vancomycin against drug resistant Staphylococcus aureus. Int J Pharm. 2012;436(1):65976. https://doi.org/10.1016/j.ijpharm.2012.07.033.

17. Singla AK, Chawla M. Chitosan: some pharmaceutical and biological aspects - an update. J Pharm Pharmacol. 2001;53(8):1047-67. https:// doi.org/10.1211/0022357011776441.

18. Nishimura K, Nishimura S, Nishi N, Saiki I, Tokura S, Azuma I. Immunological activity of chitin and its derivatives. Vaccine. 1984;2(1):93-9. https://doi.org/10.1016/S0264-410X(98)90039-1.

19. Nishimura $\mathrm{K}$, Nishimura $\mathrm{S}$-i, Nishi $\mathrm{N}$, Numata $\mathrm{F}$, Tone $\mathrm{Y}$, Tokura S, et al. Adjuvant activity of chitin derivatives in mice and guinea-pigs. Vaccine. 1985;3(5):379-84. doi:https://doi. org/10.1016/0264-410X(85)90127-6.

20. Kurita K. Chitin and chitosan: functional biopolymers from marine crustaceans. Mar Biotechnol. 2006;8(3):203. https://doi. org/10.1007/s10126-005-0097-5.

21. Sashiwa H, Kawasaki N, Nakayama A, Muraki E, Yajima H, Yamamori N, et al. Chemical modification of chitosan. Part 15: Synthesis of novel chitosan derivatives by substitution of hydrophilic amine using $\mathrm{N}$-carboxyethylchitosan ethyl ester as an intermediate. Carbohydr. Res. 2003;338(6):557-61. doi:https:// doi.org/10.1016/S0008-6215(02)00492-5.

22. Abreu FRd, Campana-Filho SP. Preparation and characterization of carboxymethylchitosan. Polímeros. 2005;15:79-83. https://doi. org/10.1590/S0104-14282005000200004.

23. Kato $\mathrm{Y}$, Onishi H, Machida Y. N-succinyl-chitosan as a drug carrier: water-insoluble and water-soluble conjugates. Biomaterials. 2004;25(5):907-15. https://doi.org/10.1016/S01429612(03)00598-2.

24. Bernkop-Schnürch A, Guggi D, Pinter Y. Thiolated chitosans: development and in vitro evaluation of a mucoadhesive, permeation enhancing oral drug delivery system. J Controlled Release. 2004;94(1):177-86. https://doi.org/10.1016/j.jconrel. 2003.10.005.

25. Roldo $\mathrm{M}$, Hornof $\mathrm{M}$, Caliceti $\mathrm{P}$, Bernkop-Schnürch A. Mucoadhesive thiolated chitosans as platforms for oral controlled drug delivery: synthesis and in vitro evaluation. Eur J Pharm Biopharm. 2004;57(1):115-21. https://doi.org/10.1016/S09396411(03)00157-7.

26. Holme KR, Perlin AS. Chitosan N-sulfate. A water-soluble polyelectrolyte Carbohydr Res. 1997;302(1):7-12. https://doi. org/10.1016/S0008-6215(97)00117-1.

27. Sadeghi AMM, Dorkoosh FA, Avadi MR, Saadat P, RafieeTehrani M, Junginger HE. Preparation, characterization and antibacterial activities of chitosan, $\mathrm{N}$-trimethyl chitosan (TMC) and $\mathrm{N}$-diethylmethyl chitosan (DEMC) nanoparticles loaded with insulin using both the ionotropic gelation and polyelectrolyte complexation methods. Int J Pharm. 2008;355(1):299-306. https:// doi.org/10.1016/j.ijpharm.2007.11.052.

28. Sobhani Z, Mohammadi Samani S, Montaseri H, Khezri E. Nanoparticles of chitosan loaded ciprofloxacin: fabrication and antimicrobial activity. Adv Pharm Bull. 2017;7(3):427-32. https:// doi.org/10.15171/apb.2017.051.

29. Regiel-Futyra A, Kus-Liśkiewicz M, Sebastian V, Irusta S, Arruebo M, Stochel G, et al. Development of noncytotoxic 
chitosan-gold nanocomposites as efficient antibacterial materials. ACS Appl Mater Interfaces. 2015;7(2):1087-99. https://doi. org/10.1021/am508094e.

30. Rampino A, Borgogna M, Blasi P, Bellich B, Cesaro A. Chitosan nanoparticles: preparation, size evolution and stability. Int $\mathbf{J}$ Pharm. 2013;455(1-2):219-28. https://doi.org/10.1016/j.ijpharm. 2013.07.034.

31. Holban AM, Grumezescu V, Grumezescu AM, Vasile BS, Trusca R, Cristescu R, et al. Antimicrobial nanospheres thin coatings prepared by advanced pulsed laser technique. Beilstein J Nanotechnol. 2014;5:872-80. https://doi.org/10.3762/bjnano.5.99.

32. Piras AM, Maisetta G, Sandreschi S, Esin S, Gazzarri M, Batoni G, et al. Preparation, physical-chemical and biological characterization of chitosan nanoparticles loaded with lysozyme. Int J Biol Macromol. 2014;67:124-31. https://doi.org/10.1016/j. ijbiomac.2014.03.016.

33. Friedman AJ, Jenny P, Schairer DO, Champer J, Qin M, Pirouz A, et al. Antimicrobial and anti-inflammatory activity of chitosan alginate nanoparticles: a targeted therapy for cutaneous pathogens. J Invest Dermatol. 2013;133(5):1231-9. https://doi.org/10.1038/ jid.2012.399.

34. de Paz LEC, Resin A, Howard KA, Sutherland DS, Wejse PL. Antimicrobial effect of chitosan nanoparticles on streptococcus mutans biofilms. Appl Environ Microbiol. 2011;77(11):3892-5. https://doi.org/10.1128/aem.02941-10.

35. Ma ZS, Lim LY. Uptake of chitosan and associated insulin in Caco- 2 cell monolayers: a comparison between chitosan molecules and chitosan nanoparticles. Pharm Res. 2003;20(11):1812-9. https://doi.org/10.1023/B:PHAM.0000003379.76417.3e.

36. Ing LY, Zin NM, Sarwar A, Katas H. Antifungal activity of chitosan nanoparticles and correlation with their physical properties. Int. J. Biomater. 2012;2012:632698. https://doi. org/10.1155/2012/632698.

37. Luo M, Jia YY, Jing ZW, Li C, Zhou SY, Mei QB, et al. Construction and optimization of $\mathrm{pH}$-sensitive nanoparticle delivery system containing PLGA and UCCs-2 for targeted treatment of Helicobacter pylori. Colloids Surf., B. 2018;164:119. https://doi.org/10.1016/j.colsurfb.2018.01.008.

38. Modi J, Joshi G, Sawant K. Chitosan based mucoadhesive nanoparticles of ketoconazole for bioavailability enhancement: formulation, optimization, in vitro and ex vivo evaluation. Drug Dev Ind Pharm. 2013;39(4):540-7. https://doi.org/10.3109/ 03639045.2012 .666978$.

39. Zhou W, Wang Y, Jian J, Song S. Self-aggregated nanoparticles based on amphiphilic poly(lactic acid)-grafted-chitosan copolymer for ocular delivery of amphotericin B. Int J Nanomed. 2013;8:3715-28. https://doi.org/10.2147/ijn.S51186.

40. Raei M, Shahidi F, Farhoodi M, Jafari SM, Rafe A. Application of whey protein-pectin nano-complex carriers for loading of lactoferrin. Int J Biol Macromol. 2017;105:281-91. https://doi. org/10.1016/j.ijbiomac.2017.07.037.

41. Huang X, Huang X, Gong Y, Xiao H, McClements DJ, Hu K. Enhancement of curcumin water dispersibility and antioxidant activity using core-shell protein-polysaccharide nanoparticles. Food Res Int. 2016;87:1-9. https://doi.org/10.1016/j.foodres. 2016.06.009.

42. Joye IJ, Nelis VA, McClements DJ. Gliadin-based nanoparticles: stabilization by post-production polysaccharide coating. Food Hydrocolloids. 2015;43:236-42. https://doi.org/10.1016/j.foodhyd. 2014.05.021.

43. Niaz T, Ihsan A, Abbasi R, Shabbir S, Noor T, Imran M. Chitosanalbumin based core shell-corona nano-antimicrobials to eradicate resistant gastric pathogen. Int J Biol Macromol. 2019;138:100618. https://doi.org/10.1016/j.ijbiomac.2019.07.165.
44. Look M, Bandyopadhyay A, Blum JS, Fahmy TM. Application of nanotechnologies for improved immune response against infectious diseases in the developing world. Adv Drug Delivery Rev. 2010;62(4):378-93. https://doi.org/10.1016/j. addr.2009.11.011.

45. Kanner E, Gorelov AV, Pechkurov D, Gorelova E, Maksimov M, Ermolaeva A. Mucosal immune system of digestive and respiratory tracts: possibilities of prevention and treatment of infectious diseases. Medical Council. 2019:100-7. https://doi. org/10.21518/2079-701X-2019-11-100-107.

46. Holmgren J, Czerkinsky C. Mucosal immunity and vaccines. Nat Med. 2005;11(4):S45-53. https://doi.org/10.1038/nm1213.

47. des Rieux A, Fievez V, Garinot M, Schneider YJ, Préat V. Nanoparticles as potential oral delivery systems of proteins and vaccines: A mechanistic approach. J. Controlled Release. 2006;116(1):1-27. https://doi.org/10.1016/j.jconrel.2006.08.013.

48. Andrianov AK, Payne LG. Polymeric carriers for oral uptake of microparticulates. Adv Drug Delivery Rev. 1998;34(2):155-70. https://doi.org/10.1016/S0169-409X(98)00038-6.

49. Illum L, Jabbal-Gill I, Hinchcliffe M, Fisher AN, Davis SS. Chitosan as a novel nasal delivery system for vaccines. Adv Drug Delivery Rev. 2001;51(1):81-96. https://doi.org/10.1016/S0169409X(01)00171-5.

50. Subbiah R, Ramalingam P, Ramasundaram S, Kim DY, Park K, Ramasamy MK, et al. N, N, N-Trimethyl chitosan nanoparticles for controlled intranasal delivery of HBV surface antigen. Carbohydr Polym. 2012;89(4):1289-97. https://doi.org/10.1016/j. carbpol.2012.04.056.

51. Julie Westerink MA, Louise Smithson S, Srivastava N, Blonder J, Coeshott C, Rosenthal GJ. ProJuvant (Pluronic F127®/chitosan) enhances the immune response to intranasally administered tetanus toxoid. Vaccine. 2001;20(5):711-23. https://doi. org/10.1016/S0264-410X(01)00423-6.

52. Phanse Y, Carrillo-Conde BR, Ramer-Tait AE, Roychoudhury R, Pohl NLB, Narasimhan B, et al. Functionalization of polyanhydride microparticles with di-mannose influences uptake by and intracellular fate within dendritic cells. Acta Biomater. 2013;9(11):8902-9. https://doi.org/10.1016/j.actbio.2013.06.024.

53. Macri C, Dumont C, Johnston AP, Mintern JD. Targeting dendritic cells: a promising strategy to improve vaccine effectiveness. Clin. Transl. Immunol. 2016;5(3):e66-e. https://doi.org/10.1038/ cti.2016.6.

54. Jung SN, Kang SK, Yeo GH, Li HY, Jiang T, Nah JW, et al. Targeted delivery of vaccine to dendritic cells by chitosan nanoparticles conjugated with a targeting peptide ligand selected by phage display technique. Macromol Biosci. 2015;15(3):395404. https://doi.org/10.1002/mabi.201400352.

55. Sun L, Song L, Zhang X, Zhou R, Yin J, Luan S. Poly $(\gamma$-glutamic acid)-based electrospun nanofibrous mats with photodynamic therapy for effectively combating wound infection. Mater Sci Eng C. 2020;113:110936. https://doi.org/10.1016/j.msec.2020.110936.

56. Dash M, Chiellini F, Ottenbrite RM, Chiellini E. Chitosan-A versatile semi-synthetic polymer in biomedical applications. Prog Polym Sci. 2011;36(8):981-1014. https://doi.org/10.1016/j. progpolymsci.2011.02.001.

57. Wu X, He C, Wu Y, Chen X, Cheng J. Nanogel-incorporated physical and chemical hybrid gels for highly effective chemo-protein combination therapy. Adv Funct Mater. 2015;25(43):6744-55. https://doi.org/10.1002/adfm.201502742.

58. Cheng Y, He C, Ding J, Xiao C, Zhuang X, Chen X. Thermosensitive hydrogels based on polypeptides for localized and sustained delivery of anticancer drugs. Biomaterials. 2013;34(38):10338-47. https://doi.org/10.1016/j.biomaterials. 2013.09.064. 
59. Park J, Kim IY, Patel M, Moon HJ, Hwang SJ, Jeong B. 2D and 3D hybrid systems for enhancement of chondrogenic differentiation of tonsil-derived mesenchymal stem cells. Adv Funct Mater. 2015;25(17):2573-82. https://doi.org/10.1002/adfm.201500299.

60. Xu M, Hao X, Hu Z, Yan Q. Palladium-bridged polymers as CO-biosignal-responsive self-healing hydrogels. Polym Chem. 2020;11(4):779-83. https://doi.org/10.1039/C9PY01660K.

61. Li Z, He C, Yuan B, Dong X, Chen X. Injectable polysaccharide hydrogels as biocompatible platforms for localized and sustained delivery of antibiotics for preventing local infections. Macromol. Biosci. 2017;17(4). https://doi.org/10.1002/mabi.201600347.

62. Hu Y, Zhang Z, Li Y, Ding X, Li D, Shen C, et al. Dual-crosslinked amorphous polysaccharide hydrogels based on chitosan/alginate for wound healing applications. Macromol Rapid Commun. 2018;39(20):1800069. https://doi.org/10.1002/marc.201800069.

63. Yang Y, Wang X, Yang F, Wang L, Wu D. Highly elastic and ultratough hybrid ionic-covalent hydrogels with tunable structures and mechanics. Adv Mater. 2018;30(18):1707071. https://doi. org/10.1002/adma.201707071.

64. Chen H, Xing X, Tan H, Jia Y, Zhou T, Chen Y, et al. Covalently antibacterial alginate-chitosan hydrogel dressing integrated gelatin microspheres containing tetracycline hydrochloride for wound healing. Mater. Sci. Eng.C. 2017;70:287-95. https://doi. org/10.1016/j.msec.2016.08.086.

65. Burkatovskaya M, Tegos GP, Swietlik E, Demidova TN, P Castano A, Hamblin MR. Use of chitosan bandage to prevent fatal infections developing from highly contaminated wounds in mice. Biomaterials. 2006;27(22):4157-64. https://doi.org/10.1016/j. biomaterials.2006.03.028.

66. Nickzad A, Déziel E. The involvement of rhamnolipids in microbial cell adhesion and biofilm development-an approach for control? Lett Appl Microbiol. 2014;58(5):447-53. https://doi. org/10.1111/lam.12211.

67. de Araujo LV, Guimaraes CR, da Silva Marquita RL, Santiago VMJ, de Souza MP, Nitschke M, et al. Rhamnolipid and surfactin: anti-adhesion/antibiofilm and antimicrobial effects. Food Control. 2016;63:171-8. https://doi.org/10.1016/j.foodcont.2015.11.036.

68. Omardien S, Brul S, Zaat SAJ. Antimicrobial activity of cationic antimicrobial peptides against gram-positives: current progress made in understanding the mode of action and the response of bacteria. Front Cell Dev Biol. 2016;4:111. https://doi.org/10.3389/ fcell.2016.00111.

69. Moussa Z, Chebl M, Patra D. Interaction of curcumin with 1,2-dioctadecanoyl-sn-glycero-3-phosphocholine liposomes: Intercalation of rhamnolipids enhances membrane fluidity, permeability and stability of drug molecule. Colloids Surf., B. 2017;149:30-7. https://doi.org/10.1016/j.colsurfb.2016.10.002.

70. Sana S, Datta S, Biswas D, Sengupta D. Assessment of synergistic antibacterial activity of combined biosurfactants revealed by bacterial cell envelop damage. Biochim Biophys Acta Biomembranes. 2018;1860(2):579-85. https://doi.org/10.1016/j. bbamem.2017.09.027.

71. Shen Z, Kuang S, Zhang Y, Yang M, Qin W, Shi X, et al. Chitosan hydrogel incorporated with dental pulp stem cell-derived exosomes alleviates periodontitis in mice via a macrophagedependent mechanism. Bioact Mater. 2020;5(4):1113-26. https:// doi.org/10.1016/j.bioactmat.2020.07.002.

72. Peracchia MT, Gref R, Minamitake Y, Domb A, Lotan N, Langer R. PEG-coated nanospheres from amphiphilic diblock and multiblock copolymers: investigation of their drug encapsulation and release characteristics. J Controlled Release. 1997;46(3):22331. https://doi.org/10.1016/s0168-3659(96)01597-0.

73. Almeida A, Silva D, Gonçalves V, Sarmento B. Synthesis and characterization of chitosan-grafted-polycaprolactone micelles for modulate intestinal paclitaxel delivery. Drug Deliv Transl Res. 2018;8(2):387-97. https://doi.org/10.1007/s13346-017-0357-8.

74. Cai Y, Leck H, Tan RW, Teo JQ, Kwa AL. Clinical experience with high-dose polymyxin $b$ against carbapenem-resistant gram-negative bacterial infections-a cohort study. Antibiotics. 2020;9(8):451. https://doi.org/10.3390/antibiotics9080451.

75. Justo JA, Bosso JA. Adverse reactions associated with systemic polymyxin therapy. Pharmacotherapy. 2015;35(1):28-33. https:// doi.org/10.1002/phar.1493.

76. Roberts KD, Azad MAK, Wang J, Horne AS, Thompson PE, Nation RL, et al. Antimicrobial activity and toxicity of the major lipopeptide components of polymyxin $\mathrm{b}$ and colistin: last-line antibiotics against multidrug-resistant gram-negative bacteria. ACS Infect Dis. 2015;1(11):568-75. https://doi.org/10.1021/ acsinfecdis. 5 b00085.

77. Wallace SJ, Li J, Nation RL, Prankerd RJ, Velkov T, Boyd BJ. Self-assembly behavior of colistin and its prodrug colistin methanesulfonate: implications for solution stability and solubilization. J Phys Chem B. 2010;114(14):4836-40. https:// doi.org/10.1021/jp100458x.

78. Zhu C, Schneider EK, Wang J, Kempe K, Wilson P, Velkov T, et al. A traceless reversible polymeric colistin prodrug to combat multidrug-resistant (MDR) gram-negative bacteria. J Controlled Release. 2017;259:83-91. https://doi.org/10.1016/j.jconrel. 2017.02.005.

79. Alipour M, Suntres ZE. Liposomal antibiotic formulations for targeting the lungs in the treatment of Pseudomonas aeruginosa. Ther Deliv. 2014;5(4):409-27. https://doi.org/10.4155/tde.14.13.

80. Gounani Z, Asadollahi MA, Meyer RL, Arpanaei A. Loading of polymyxin B onto anionic mesoporous silica nanoparticles retains antibacterial activity and enhances biocompatibility. Int J Pharm. 2018;537(1):148-61. https://doi.org/10.1016/j.ijpharm. 2017.12.039.

81. Obuobi S, Voo ZX, Low MW, Czarny B, Selvarajan V, Ibrahim $\mathrm{NL}$, et al. Phenylboronic acid functionalized polycarbonate hydrogels for controlled release of polymyxin $b$ in pseudomonas aeruginosa infected burn wounds. Adv Healthcare Mater. 2018;7(13):1701388. https://doi.org/10.1002/adhm.201701388.

82. Liu YH, Kuo SC, Yao BY, Fang ZS, Lee YT, Chang YC, et al. Colistin nanoparticle assembly by coacervate complexation with polyanionic peptides for treating drug-resistant gramnegative bacteria. Acta Biomater. 2018;82:133-42. https://doi. org/10.1016/j.actbio.2018.10.013.

83. Chai M, Gao Y, Liu J, Deng Y, Hu D, Jin Q, et al. Polymyxin B-polysaccharide polyion nanocomplex with improved biocompatibility and unaffected antibacterial activity for acute lung infection management. Adv. Healthcare Mater. 2020;9(3). https://doi.org/10.1002/adhm.201901542.

84. El-Sharif AA, Hussain MHM. Chitosan-EDTA new combination is a promising candidate for treatment of bacterial and fungal infections. Curr Microbiol. 2011;62(3):739-45. https://doi. org/10.1007/s00284-010-9777-0.

85. Camacho-Alonso F, Julián-Belmonte E, Chiva-García F, Martínez-Beneyto Y. Bactericidal efficacy of photodynamic therapy and chitosan in root canals experimentally infected with enterococcus faecalis: an in vitro study. Photomed Laser Surg. 2017;35(4):184-9. https://doi.org/10.1089/pho.2016.4148.

86. Tesfamariam B. Bioresorbable vascular scaffolds: biodegradation, drug delivery and vascular remodeling. Pharmacol Res. 2016;107:163-71. https://doi.org/10.1016/j.phrs.2016.03.020.

87. Jinnouchi H, Torii S, Sakamoto A, Kolodgie FD, Virmani R, Finn AV. Fully bioresorbable vascular scaffolds: lessons learned and future directions. Nat Rev Cardiol. 2019;16(5):286-304. https:// doi.org/10.1038/s41569-018-0124-7. 
88. Wei H, Yan S, Goel S, Menary G. Characterization and modelling the mechanical behaviour of poly(l-lactic acid) for the manufacture of bioresorbable vascular scaffolds by stretch blow moulding. Int J Mater Form. 2020;13(1):43-57. https://doi.org/10.1007/s12289018-01463-2.

89. Khalifehzadeh R, Ciridon W, Ratner BD. Surface fluorination of polylactide as a path to improve platelet associated hemocompatibility. Acta Biomater. 2018;78:23-35. https://doi. org/10.1016/j.actbio.2018.07.042.
90. Yu Y, Cui R, Wang X, Yang H, Li H. Preparation of multifunctional poly(L-lactic acid) film using heparin-mimetic polysaccharide multilayers: hemocompatibility, cytotoxicity, antibacterial and drug loading/releasing properties. Int $\mathbf{J}$ Biol Macromol. 2020;155:14-26. https://doi.org/10.1016/j. ijbiomac.2020.03.180.

Publisher's Note Springer Nature remains neutral with regard to jurisdictional claims in published maps and institutional affiliations. 